\title{
Use of Radiation From Incandescent Particles as an Indication of Flame Temperature
}

\author{
Earle K. Plyler and Curtis J. Humphreys
}

\begin{abstract}
This study was undertaken to determine if the radiation from incandescent carbon particles could be utilized in the measurement of the temperature of flames. This is a preliminary investigation of the measurement of flame temperatures by using the intensities in the ultraviolet continuum at two wavelengths chosen in regions comparatively free from molecular bands and comparing these energies with the continuous emission at the same wavelengths of a calibrated tungsten ribbon lamp containing a quartz window. Fuel-rich oxyacetylene flames were used because they contain the desired incandescent particles. The ratios of intensities in the chosen spectral regions were determined photographically and photoelectrically. Interpretation of the observed ratio for a given flame, in terms of temperature has been made, and flame temperatures from $1,900^{\circ}$ to $2,400^{\circ} \mathrm{C}$ have been determined.
\end{abstract}

\section{Introduction}

The temperature of a flame is something extremely difficult to define. This should become evident when it is pointed out that the energy liberated in the combustion process appears in many forms and that at a given instant a state of equilibrium can hardly be expected to be realized. Thus, the molecules formed as products of combustion will have varying amounts of translational, vibrational, or rotational energy, each form of energy leading to a corresponding temperature. A detailed discussion of flame temperatures and of several methods of measuring them is to be found in a book by Gaydon [1]. ${ }^{2}$ The apparent temperature of a flame will necessarily depend on the method of measurement chosen, because in general the method will utilize a physical process corresponding to a characteristic temperature. Several methods for the measurement of flame temperatures have been devised or proposed. For flame temperatures below $1,700^{\circ} \mathrm{C}$ the problem is relatively simple because high melting-point temperature detectors can be inserted directly into the flame. For higher temperatures some method utilizing radiant energy must be emploved. Some of the methods are line reversal, generally by using sodium vapor, and determinations based on intensity ratios among the components of the emission bands. The lastnamed method leads to what may be called the rotational temperature.

The present study is concerned with a special class of flames, namely, opaque or semiopaque flames. The appearance of such a flame depends on the abundance of incandescent particles in the flame, which may be either carbon particles originating in the combustion process or solid particles of some other material purposely introduced. For practical purposes the radiant energy emitted by a cloud of glowing particles may be regarded as having properties similar to those of the spectral continuum from the surface of an incandescent body at the same

${ }^{1}$ A partial report of this work was given at the New York meeting of the Optical Society of America (March 1949). J. Opt. Soc. Am. 39, 639 (1949).

2 Figures in brackets indicate the literature references at the end of this paper. temperature. The essential difference is that the over-all intensity, or total radiant flux, will depend on the number of particles present and will always be less than that from the surface of a solid or liquid at the same temperature.

The spectral distribution of energy from a hot body emitting continuously is a function of temperature. If the emitter is a black body, this temperature dependence may be expressed by any of a number of well-known formulas [2], the Planck distribution law being the most precise. The form of this distribution curve, in which energies are plotted as ordinates and wavelengths as abscissas, is somewhat similar to a probability curve. At any given temperature there is a maximum of energy emission corresponding to some wavelength. The maximum of emission shifts to shorter wavelengths for higher temperatures according to the well-known Wien displacement law, $\lambda T=K$, where $\lambda$ is the wavelength of maximum emission and $T$ the absolute temperature. It is obvious that if the wavelength position of the emission maximum could be located precisely, the corresponding absolute temperature would be determined. Unfortunately, the precise location of the emission maximum is beset with several experimental difficulties, not the least of which is that it is broad and not too well defined. Another method of temperature measurement is to establish the form of the energy distribution curve. This may be accompplished by determining the relative values of energy emitted at a number of wavelengths. When visible light is employed, this is the procedure used in determining what is called the color temperature $[2$, p. 23 and 382]. The method depends on the comparison of intensities of different portions of the continuous spectrum, as isolated by color filters. Its application to flame-temperature determinations is discussed by Gaydon [1, p. 175]. The temperature measured is that of the glowing particles in the flame, which may not be completely in equilibrium with the flame gases. Emission characteristics of molecules excited in the combustion process is a possible source of error, which can be avoided by 
using filters that transmit only in wavelength regions free of these characteristic spectra.

The representation of spectral energy distribution from continuous emitters by a precise formula is ordinarily restricted to black bodies. Corrections, depending on variations in emissivity, necessary to interpreting spectral distributions encountered in actual glowing bodies in terms of temperature, present no great difficulty.

For this study of flame temperatures, the ultraviolet region was selected because: (1) there is no appreciable radiation from surrounding objects that have temperatures below $1,600^{\circ} \mathrm{C}$, (2) the energy emitted increases more rapidly with temperature than it does in the infrared, and (3) the difference between true and brightness temperature is less than in the infrared [2, p. 380].

Although the procedures for measuring color temperatures are well known, very little work has been reported previously on the measurement of flame temperatures by establishment of the energy distribution in the ultraviolet.

\section{Experimental Procedure}

Oxyacetylene flames were selected for use in these experiments, a choice based on the availability of equipment for producing and controling such flames and the fact that rich mixtures of oxygen and acetylene burn with an opaque flame owing to the large amounts of carbon liberated, a condition favoring this method. The essential units of a commercial welding outfit, consisting of a torch with a number 4 welding tip, regulators for the respective gases, and gas cylinders, were used to produce the flames. Both the volume of the flame and the relative amounts of the two gases are controllable by manipulation of the valves in the mixing unit of the torch and finding the appropriate settings of the needle valves in the regulators. Rotameters were used in the later stages of the work in order to measure the rates of flow of the gases. The appearance of the flame varies greatly with the mixture ratio. When pure acetylene is burned it is yellow and sooty. With small amounts of oxygen it is a brilliant white. As the oxygen flow is increased the white appearance thins out, and the base of the flame becomes blue in color. Further additions of oxygen are accompanied by a complete disappearance of the white color, the flame becoming blue and essentially transparent throughout.

A ribbon-filament lamp was used as the reference temperature standard. These lamps have a heavyduty bipost base, a silica window attached to a side arm by a graded seal, and a maximum current rating of about 40 amperes. A calibration of the lamp used was furnished by the Bureau's pyrometry laboratory. This consisted of values of filament current corresponding to brightness temperatures at 100-deg intervals from $1,900^{\circ}$ to $2,400^{\circ} \mathrm{C}$. Following the usual pyrometric practice these brightness temperatures were measured at a wave length of $0.65 \mu$. The uncertainty of the calibration at $2,400^{\circ} \mathrm{C}$ was estimated at $7 \mathrm{deg} \mathrm{C}$. The highest temperature included in the series of calibrated points represents the highest safe operating temperature of the lamp. Oxyacetylene flames giving temperatures between these limits are essentially opaque, since they occur for mixtures relatively rich in fuel.

The practical application of the method of measuring temperatures herein described requires the development of a simple portable radiometric device. The basic component around which the device was actually built was an RCA 935 phototube. Since the temperature evaluation depends on the determination of the form of the spectral energy curve, it is desirable to obtain readings at three wavelengths. Readings at two wavelengths separated by an interval of a few hundred Angstroms are, however, sufficient to establish the slope of the curve over the interval, and provide the basis for a temperature calibration.

In order to isolate two selected wavelength regions, two narrow-band-pass filters were prepared for use with the phototube. Each consisted of a combination of an interference filter with a glass filter. The interference filter, which is essentially a Fabry-Perot interferometer with a fixed separation, has a narrow transmission band at a specified wavelength. There are, however, secondary maxima at longer and shorter wavelengths. These may be eliminated by combining the interference filters with a glass filter, which isolates the region in which the principal maximum of the interference filter occurs. The following combinations were adopted: interference filter having a transmission maximum at 3541 A, with a Corning glass filter, code number 9863; interference filter, transmission maximum $4140 \mathrm{~A}$, with a Schott filter designated BG28. These filters were selected after examination of flame spectrograms showed that the spectrum was sensibly free of characteristic molecular bands at the wavelengths coincident with the transmission maxima of the filters. The measurements were thus confined to the continuous emission originating in the glowing particles in the flame.

A small housing was constructed to serve as a light shield and permit placing the filters against a window opening in front of the cell cathodes. The remaining components of the metering device consisted of a balanced d-c amplifier described in an earlier publication [3] and a galvonometer and resistance network to vary the sensitivity.

The performance and reproducibility of readings of the phototube meter were tested by a series of exposures to the ribbon-filament lamp. Readings were taken with each filter combination in place for each temperature setting between $1,900^{\circ}$ and $2,400^{\circ} \mathrm{C}$. The results of this test are displayed in figure 1. Two of the curves are plots of deflections corresponding to photocell readings for the different temperatures for the respective filters. The third curve represents the ratio of deflections at each wavelength for the two filters. The variation of this ratio with temperature provides the basis for a calibration of the meter for temperature readings. The data represented in figure 1 are for one set of observations rather than for 


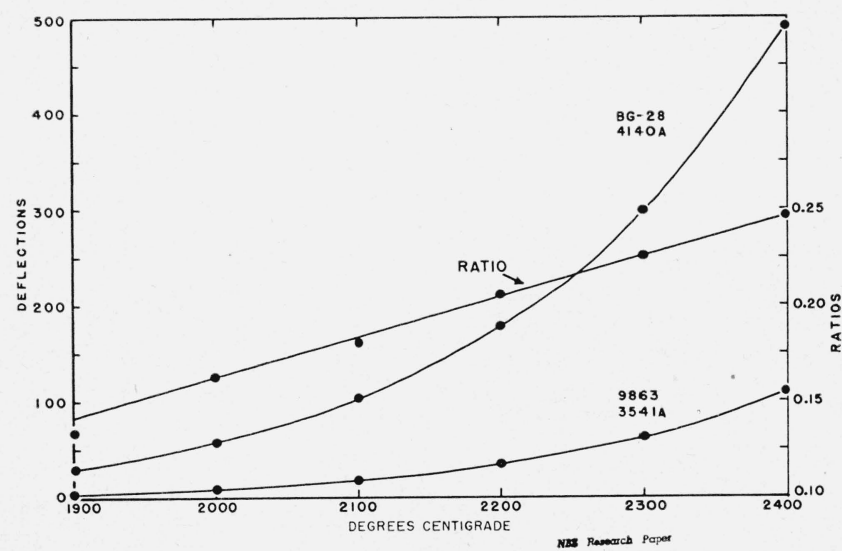

FIGURE 1. Filier readings for different temperatures and ratios of filter readings plotted against temperatures of ribbon filament tungsten lamp.

an average of several. It is to be noted that the curves are extremely regular. The rapid increase in deflections with temperature, shown by the curves representing filter readings, indicates that radiant energy in the ultraviolet provides a sensitive scale of temperature. If one had been measuring temperatures lower than $1,900^{\circ} \mathrm{C}$, it would have been advantageous to select two wavelengths in the visible or infrared in order to have more radiant energy available.

A series of observations were made on the flame using the same filters as were employed for the tungsten ribbon lamp. The flame was changed by using a constant amount of $\mathrm{C}_{2} \mathrm{H}_{2}$ and varying the $\mathrm{O}_{2}$ content of the mixture. In figure 2 are shown the ratios of the radiant energy emitted through the filter at $3541 \mathrm{~A}$ to that emitted at $4140 \mathrm{~A}$ for different amounts of oxygen. All the observed ratios fall on a smooth curve except for the fourth determination. Occasionally the flame becomes unsteady and some variation in the ratio results. The conditions for the reproducibility of the flame will be discussed later. When the temperatures for the six points in figure 2 are determined from the calibration curve of figure 1 , it is found that they start at about $1,850^{\circ} \mathrm{C}$ for the first point and reach a value of $2,500^{\circ} \mathrm{C}$ for the sixth point.

In order to have another method of measuring the ratios of emitted energies at 3541 and $4140 \mathrm{~A}$, the spectrographic method was employed. At the time of measuring the radiant energy with the photocell, exposures were made of the flame spectra by the use of a Hilger E2 spectrograph. On evaluation of the plates it was found that the temperature of the second point of figure 2 was $1,950^{\circ} \mathrm{C}$, the third close to $2,000^{\circ}$, the fourth $2,100^{\circ}$, the fifth, $2,300^{\circ}$, and the sixth somewhat above $2,400^{\circ}$.

The method of evaluation of flame temperature by the spectrograms is illustrated in figure 3 which shows a set of spectra of the flame and the filament with comparison spectra of the mercury arc. The lines, reading from the left, at $2537,2804,3132,3650$, and $4358 \mathrm{~A}$ are from an $\mathrm{Hg}$ arc and are used for the wave-

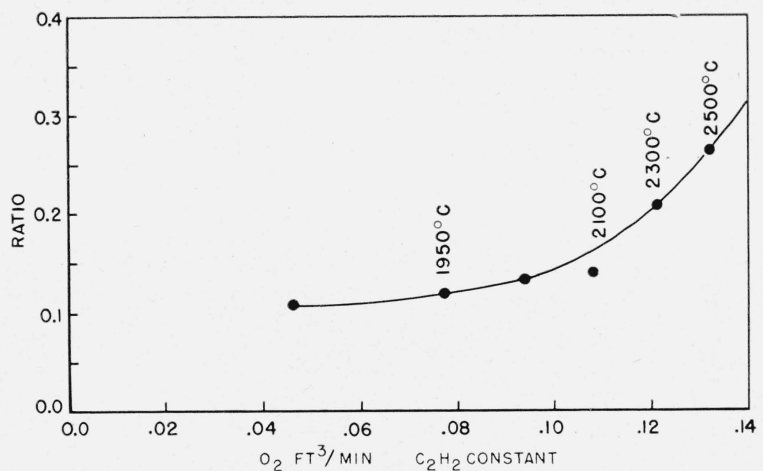

Figure 2. Ratios of filter readings compared with oxygen comsumption.

Temperature equivalents noted.

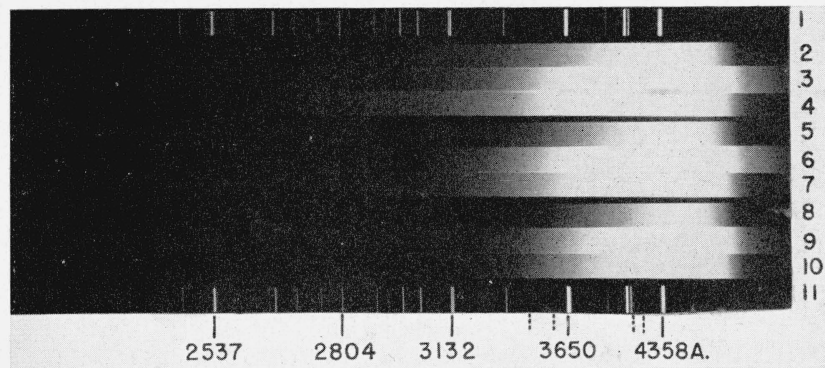

FIGURE 3. Spectrogram showing three flame spectra compared with filament exposures at 2,400 $, 2,100^{\circ}$, and $1,900^{\circ} \mathrm{C}$.

Exposures 1 and 11-mercury arc, five lines identified by wavelengths. Regions transmitted by the two filters designated by dotted lines. Exposures 2 and 4 -continuous spectrum Exposure 3 5 and 7 -ribbon filament, $T=2,100$, exposures 3 and 12 sec. Exposure 6 -bright opaque flame, exposure 4 minutes. Exposures 8 and 10 -ribbon filament,
$T=1,900$, exposures 5 and 18 sec. Exposure 9 -yellowish opaque flame, exposure 4 minutes.

length determination of regions in the flame spectra. The spectra are displayed in groups of three. The flame spectrum under selected operating conditions is at the center of each group and is flanked on either side by filament exposures at the same temperature but with different length of exposure. The three flame spectra were produced under three different operating conditions, and are compared with filament exposures at $2,400,2,100$, and $1,900^{\circ} \mathrm{C}$, respectively, reading from top to bottom on the plate. The filament exposures adjacent to each flame spectrum are in the ratio of 1 to 4 . The highest filament temperature is clearly higher than the highest flame temperature recorded on the plate. For the lowest temperature the match is fairly close. The match is good for the intermediate temperature when the longest filament exposure is compared. With some experience it is possible to obtain much better matches than shown on the plate. Only the continuous portions of the flame spectra are considered in making these comparisons.

The matching of intensities was carried out by visual examination only, this being considered satisfactory in view of the limited objectives of the investigation. An alternate method that certainly would 


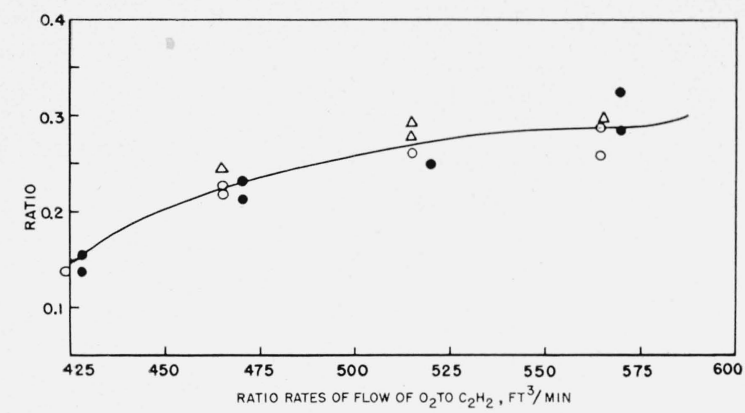

Figure 4. Composite curve, filter ratios versus ratios of fuel consumption with rotameters controling flow of both gases.

Three types of symbols refer to three observations.

be more precise because it is entirely objective would be to determine the plate densities with a densitometer for both the flame and filament temperatures and consider the criterion of equal temperatures to be a constant ratio of the intensities between two wavelengths for the flame and for the lamp. However, this process of matching exposures on photographic plates by a spectroscopic method, which is tedious and detailed if carried out precisely, is not required for temperature measurements, but was used as a check on the photocell method.

The radiation from the part of the flame that was 2 in. above the tip of the torch, was focused on the photocell so that the results from separate trials could be compared. Figure 4 shows a plot of the ratios of the energies for different fuel mixtures as a function of the fuel mixture. The composite curve is intended to be the closest possible representation of the observations taken on several different days, each point in the graph corresponding to an observation. The same symbol is used to distinguish observations on a given day. The spread of the points at any one fuel ratio is produced largely by imperfect duplication of the fuel mixture for the separate determinations. A small change in the fuel ratio would change the ratio of energies by several percent. A smooth curve drawn through these points would give an arbitrary scale of temperature. This curve would also make it possible to operate the flame under the same condition at different times.

The temperatures estimated on the basis of the observed ratios and the calibration curve of figure 1 are in terms of the brightness temperatures of the filament of the tungsten lamp. The true temperature of the filament as calculated from the Wien equation in degrees Kelvin corresponding to $2,400^{\circ}$ $\mathrm{C}$ brightness temperature is about $2,900^{\circ} \mathrm{K}$. If an emissivity of 0.7 is assumed for the particles in the ultraviolet they would have a true temperature of $2,830^{\circ} \mathrm{K}$ when the brightness temperature of the filament is matched, and with the emissivity of 0.8 the true temperature would be $2,810^{\circ} \mathrm{K}$. With almost equal fuel ratios the $\mathrm{OH}$ spectrum of the oxyacetylene flame has been recently measured $\lceil 4\rceil$. It was found that the $\mathrm{OH}$ vibrational and rotational temperatures corresponded to $2,600^{\circ} \mathrm{K}$ with possible error being up to $200^{\circ}$. These results indicate that the temperature determined by the solid particles is in satisfactory agreement with the $\mathrm{OH}$ temperature determination. Also, the spectrum of $\mathrm{C}_{2}$ emitted by an oxyacetylene flame has been observed by the use of a grating spectrometer in the near infrared $\lceil 5\rceil$. The temperature deduced from the rotational spectra was of the order of $2,700^{\circ} \mathrm{K}$. This value is in good agreement with results obtained on the emission of radiant energy by solid particles as found in the present work.

\section{Discussion of Results and Evaluation of Corrections}

Up to this point in the description of these experiments no discussion has been introduced regarding the limitations on temperature matching at different wavelengths when nonblack bodies are being compared. It is apparent that a change of emissivity with wavelength will change the form of the spectral energy distribution curve and thus prevent coincidence of curves from different sources, except for very limited ranges. The change of emissivity with wavelength usually proceeds at a very slow rate so that mismatch from this cause is slight over the range of wavelengths used in these experiments. It is to be noted also that, if one is comparing different radiators each with constant but different emissivities or if one is comparing one such radiator with a black body where the emissivity is always unity, it is still impossible to get an exact energy match at two wavelengths. This is a consequence of the following equation based on the Wien distribution formula,

$$
\frac{1}{T}-\frac{1}{S_{\lambda}}=\frac{\lambda \log e_{\lambda}}{C_{2} \log e}
$$

where $T$ is the true temperature, $S_{\lambda}$ is the brightness temperature at wavelength $\lambda, C_{2}$ the radiation constant, and $e_{\lambda}$ the spectral emissivity. A conversion by means of this equation of the calibrated values of brightness temperature of the ribbon-filament lamp, at $0.6650 \mu$, to the corresponding values of 0.4140 and $0.3541 \mu$, based on accepted vaiues of emissivity of tungsten has been made. The procedure is to calculate the true temperatures corresponding to the calibrated values of brightness temperature, then taking these true temperatures as the initial data, substitute in the equation to obtain the brightness temperatures at the wavelengths of the respective filter transmissions. The following typical example is selected to illustrate the mismatch at these wavelengths. Given a true temperature of $2,713^{\circ} \mathrm{C}$, the brightness temperature of the filament is $2,520^{\circ} \mathrm{C}$ at $\lambda=0.4140 \mu$ and $2,547^{\circ} \mathrm{C}$ for $\lambda=0.3541 \mu$, when $e_{\lambda}=0.45$. The consequence of this is that, in comparing the intensity of the lamp with that of a black body, if the energies correspond at $0.4140 \mu$, the energy would be greater for the lamp at $0.3541 \mu$ and make it appear brighter by $27 \mathrm{deg}$ C. This difference is less than the experimental error when observations are made on the 
flame. It can be shown from eq 1 that the difference between the true and the brightness temperatures decreases in the ultraviolet region as compared with the visible spectrum. When the emissivity increases, the difference between the true and the brightness temperatures becomes less. While the emissivity of carbon particles is not well known at high temperatures, W. E. Forsythe [2, p. 380] gives the following values for the emissivity of carbon: $e_{\lambda}(\lambda=0.465 \mu)$, at $T=1,200^{\circ} \mathrm{K}$, is 0.86 , and, at $T=2,200^{\circ} \mathrm{K}$, is 0.79 .

Calculations have been made on the temperature of the particles on the basis of $e_{\lambda}$ being equal to 0.7 and 0.8 . When the brightness temperature is $2,547^{\circ}$ $\mathrm{C}$ the true temperatures at a wavelength of $0.354 \mu$ are $2,618^{\circ}$ and $2,591^{\circ} \mathrm{C}$ for emissivities of 0.7 and 0.8 , respectively. If the emissivity of the particles were 0.45 instead of 0.7 at $0.354 \mu$, the true temperature would be $95 \mathrm{deg}$ higher for the same brightness temperature. Until more accurate values of emissivity are known there will probably be an uncertainty in the estimates of temperature of about $100 \mathrm{deg}$ C. This is of the same order of magnitude as the errors introduced by the observed ratios of intensities.

Flame temperatures determined by this method are in essential agreement with temperatures deduced from spectroscopic observations of flames produced under similar conditions.

\section{References}

[1] A. G. Gaydon, Spectroscopy and combustion theory (Chapman \& Hall, London, 1948).

[2] W. E. Forsythe, Measurement of radiant energy, chapter 1 (McGraw-Hill Book Co., Inc., New York, N. Y., 1937).

[3] W. W. Coblentz and R. Stair, A portable ultraviolet intensity meter, consisting of a balanced amplifier, photoelectric cell, and microammeter, BS J. Research 12, 231 (1934) RP647.

[4] W. S. Benedict, E. K. Plyler, and C. J. Humphreys, Vibration-rotation lines of $\mathrm{OH}$ in flame spectra, Bul. Am. Phys. Soc. 26, 50 (1951).

[5] W. S. Benedict and Earle K. Plyler, Bands of $\mathrm{C}_{2}$ and $\mathrm{CN}$ in infrared flame spectra, Bul. Am. Phys. Soc. 26, 54 (1951).

Washington, August 29, 1950. 